\title{
Characteristics of the patients followed with the diagnosis of common variable immunodeficiency and the complications
}

\author{
SEMIHA BAHCECI ERDEM ${ }^{I}$, NESRIN GULEZI, FERAH GENEL ${ }^{I}$, SAIT KARAMAN ${ }^{1}$, \\ HIKMET T. NACAROGLU ${ }^{2}$
}

'Department of Pediatric Allergy and Immunology, Dr. Behçet Uz Children Disease and Surgery Training and Research Hospital, Izmir, Turkey

${ }^{2}$ Department of Pediatric Allergy and Immunology, Istambul Medipol University, Istambul, Turkey

\begin{abstract}
Introduction: In this study, we aimed to retrospectively evaluate the clinical and laboratory findings and complications of 28 common variable immunodeficiency (CVID) patients.

Material and methods: The clinical features and laboratory data of 28 CVID patients were evaluated.

Results: Nineteen patients were male. In 53.5\% of the cases, complications included inflammatory bowel disease, cytopenia, bronchiectasis, granulomatous lymphocytic interstitial lung disease (ILD) and asthma. In their immunological evaluations, IgG, IgM, and IgA mean values were $474.8 \pm 214.1 \mathrm{mg} / \mathrm{dl}$; $56.7 \pm 41.9 \mathrm{mg} / \mathrm{dl} ; 35.3 \pm 58.2 \mathrm{mg} / \mathrm{dl}$, respectively, and the vaccine response was positive in $64.2 \%$ of the cases. In all age groups, absolute lymphocyte counts, naive (CD19+IgD+27-), nonswitch $(C D 19+I g D-27+)$ memory $B$ cells were numerically higher when compared to the data of healthy children; however, although switch memory $(C D 19+I g D+27+) B$ cells were proportionally low in the 4-8 and 12-18 age groups, they were low both numerically and proportionally in the 8-12 age group. No statistically significant difference was found between the cases with complications and without complications. But the cases with pulmonary complications were compared within the group, the CD8 ratio was high but the IgA level was low in patients with bronchiectasis and CD3 was numerically and proportionally low in the cases with ILD compared to others. According to the Paris classification, 11/27 (40.7\%) of the cases, $3 / 27(11.1 \%)$ of them and 13/27 (48.2\%) of them were evaluated as $M B 0, M B 1$, and $M B 2$, respectively.

Conclusions: In genetic studies, TACI (trans-membrane activator and calcium-modulating cyclophilin ligand interactor - TNFRSF13B) mutation was found positive in $25 \%$ of the cases.
\end{abstract}

Key words: primary immunodeficiency, hypogammaglobulinemia, common variable immunodeficiency, TACI.

(Centr Eur J Immunol 2019; 44 (2): 119-126)

\section{Introduction}

Common variable immunodeficiency (CVID) is the most common antibody deficiency which can develop due to failure in immunoglobulin and protective antibody production, which is generally characterized by an increased tendency to frequent infections caused by bacterial agents, autoimmunity, and malignancy, and provides clinical findings [1-5]. Its prevalence varies between 1/10,000 and 1/100,000 [1, 4, 6-9]. The recurrent infections of the respiratory tract may specially lead to irreversible damages of the lungs such as bronchiectasis and chronic lung disease. Except for infection, lymphoid hyperplasia, gastrointestinal inflammation and autoimmune diseases (immune thrombocytopenia, autoimmune hemolytic anemia, Hashimoto's thyroiditis, atrophic gastritis, pernicious anemia, collagen vascular diseases, etc.), malignancy (especially lymphoma) can be observed both as a presenting symptom and a complication in patients as a result of abnormal $\mathrm{T}$ cell function and immunodysregulation [10]. The European Society for Immunodeficiencies criteria (www.ESID.org) are widely used in the diagnosis [11]. In the laboratory evaluation of CVID patients, the absolute lymphocyte count is normal, and at least 2 major immunoglobulin levels (especially the level of $\operatorname{IgG}$ and $\operatorname{IgA}$ and/or IgM) are below 2 SD of the levels determined by age. The antibody response to vaccines is usually damaged $[1,2]$. $\mathrm{B}$ cells are numerically and proportionally normal or close to normal. Only about $20 \%$ of the patients have a low B cell count, and this low ratio indicates a poor prognosis in patients. Among B cells, especially memory B cells are very important

Correspondence: Hikmet T. Nacaroglu, MD, Department of Pediatric Allergy and Immunology, Dr. Behçet Uz Children Disease and Surgery Training and Research Hospital, Alsancak/Izmir, 35220 Izmir, Turkey, e-mail: tekin212@gmail.com Submitted: 9.12.2016, Accepted: 14.11.2017 
in CVID immunopathogenesis [12]. Numerical or functional reduction, disruption in cytokine production, a decrease in T-helper functions, disruption in signal transduction, disruption in CD40 ligand expression, an increase in suppressor T cell functions, and a decrease in NK cell count can be observed in T cells. Although CVID is observed sporadically in many patients, it is inherited familial and mostly autosomal dominant and less autosomal recessive in $10-25 \%$ of the patients [1, 2 , $13,14]$. Different monogenic defects that specifically lead to the CVID phenotype have been reported in recent years. These defects are inducible co-stimulator molecule (ICOS), TACI (trans-membrane activator and calcium-modulating cyclophilin ligand interactor - TNFRSF13B), BAFF-R (B cell activating factor-receptor TNFRSF13C), CD19, CD20, CD21, CD81, lipopolysaccharide ligand interactor (LRBA) and MSH5 (MutS homolog 5) $[6,15,16]$. These defects lead to the impairment of the functions of molecules that play a critical role and are responsible for the coordination of many complex pathways responsible for the formation of the effective humoral response.

The basic treatment is to replace $\mathrm{IgG}$ at sufficient doses. The aim is to protect against life-threatening serious bacterial infections or to reduce the frequency of these infections. The recent developments in the management of the disease, regular intravenous immunoglobulin use and the changes in antimicrobial treatments have extended the patients' length of life. Thus, non-infectious complications have begun to be further noticed. It has become possible to evaluate the clinical correlations of the differences in immunopathogenesis along with the detection of various complications and the addition of molecular genetic findings.

Table 1. Demographic data of the patients $(N=28)$

\begin{tabular}{|c|c|c|c|}
\hline Data & $n$ & \multicolumn{2}{|c|}{ Percent } \\
\hline \multicolumn{4}{|l|}{ Gender } \\
\hline Female & 9 & 32 & \\
\hline \multirow[t]{2}{*}{ Male } & 19 & 68 & \\
\hline & $\begin{array}{c}\text { Mean } \pm \text { SD } \\
\text { (month) }\end{array}$ & $\begin{array}{c}\text { Min-max } \\
\text { (month) }\end{array}$ & $\begin{array}{l}\text { Median } \\
\text { (month) }\end{array}$ \\
\hline Age & $112.8 \pm 61.2$ & $25.2-261.6$ & 96 \\
\hline Symptom age & $45.6 \pm 45.6$ & $3.6-112.8$ & 36 \\
\hline Diagnosis age & $80.4 \pm 44.4$ & $12-180$ & 70.8 \\
\hline \multirow[t]{2}{*}{ Duration of follow-up } & $3.31 \pm 2.9$ & $3.6-108$ & 24 \\
\hline & $n$ & Percent & \\
\hline \multicolumn{4}{|l|}{ Parental kinship } \\
\hline Yes & 10 & 35.7 & \\
\hline No & 18 & 64.3 & \\
\hline \multicolumn{4}{|l|}{ PID history in the family } \\
\hline Yes & 3 & 10.7 & \\
\hline No & 25 & 89.3 & \\
\hline
\end{tabular}

Here, we aimed to investigate the characteristics of the patients followed with the diagnosis of CVID and the complications developed in our patients.

\section{Material and methods}

The clinical features, laboratory data: immunoglobulin levels, absolute lymphocyte counts and sub-groups, vaccine responses (anti-tetanus toxoid antibody, anti-Haemophilus influenzae type B antibody - antiHIB, hepatitis B surface antibody - antiHBs, anti-diphtheria toxin antibody), naive, non-switch memory and switch memory B cells and the complications that developed during follow-up and TACI gene mutation analyses of 28 patients who were admitted to our hospital's Department of Immunology in the years 20032014 and who were followed with the diagnosis of CVID according to the European Society for Immunodeficiencies criteria (www.ESID.org) were evaluated retrospectively in the study. Pulmonary complications were classified as asthma, interstitial lung disease (ILD) and bronchiectasis [17]. Flow-cytometric analyses were performed by Beckman-Coulter 4-color FACS analysis program, and they were compared with 23 healthy control data of the appropriate age group. Immunoglobulin values (SIEMENS, Germany) were studied with the nephelometric method in the BN system in the plasma, and they were compared with the literature data of healthy Turkish aged-matched children [18]. The vaccine responses (antiHIB, antiHBs, antitetanus) were analyzed by Enzyme-Linked Immunosorbent Assay (ELISA) method. The TACI mutation analyses of the cases were performed in a genetic laboratory outside of our hospital. The study was approved by the Ethics Committee of Dr. Behçet Uz Children's Hospital (Decision No. 2015/42).

\section{Statistical analysis}

Numerical data were recorded as mean \pm SD. Statistical comparison of the data was made in SPSS 18 program using Pearson's, Spearman, $\chi^{2}$ and correlation analyses, Mann-Whitney $\mathrm{U}$, independent sample $t$-tests; $p$ values less than 0.05 were considered as statistically significant.

\section{Results}

The male to female ratio of the cases is $19 / 9$, and their demographic data, family history and parental kinship ratios, the age at diagnosis, symptom ages, and follow-up durations are presented in Table 1. When the admission findings are examined, the frequent infection was the presenting symptom in all cases. While $88 \%$ of these infections were sinopulmonary infections, this infection was accompanied by diarrhea in $12 \%$ of them. In their immunological evaluations, $\operatorname{IgG}$ was $481.8 \pm 213.2 \mathrm{mg} / \mathrm{dl} ;$ IgA was $39.2 \pm 59.4 \mathrm{mg} / \mathrm{dl} ;$ IgM was $52.5 \pm 41.3 \mathrm{mg} / \mathrm{dl}$ and vaccine response (anti-tetanus toxoid antibody and antiHbs) was positive in $64.2 \%(n=18)$ of the 
cases. CD3, CD4, CD8 and NK cells in the lymphocyte subgroups were numerically and proportionally within normal limits. Naive and memory B cells could be analyzed in 27 cases. Accordingly, in all age groups, the absolute lymphocyte counts, naive (CD19+IgD+27-), nonswitch (CD19+IgD-27+), memory $\mathrm{B}$ cells were numerically higher when compared to the data of healthy children; however, although switch memory (CD19+IgD+27+) B cells were numerically high but proportionally low in the 4-8 and 12-18 age groups, they were found to be low both numerically and proportionally in the 8-12 age group. In the patient group, there was a statistically significant increase in the CD19 ratio and nonswitch B-cell ratio and counts in the 4-8 age group, and in the CD19 B-cell count in the 8-12 age group (Tables $2 \mathrm{~A}$ and 2A). According to the Paris classification, $11 / 27(40.7 \%)$ cases, $3 / 27(11.1 \%)$ of them and $13 / 27(48.2 \%)$ of them were evaluated as MB0, MB1, and
MB2, respectively [19]. TACI mutation was studied in all cases, it was found positive in $25 \%(n=7)$ of the cases including heterozygote in 5 cases $(17.8 \%)$ and homozygote in 2 cases (7.2\%). The complication was found in $53.5 \%(15 / 28)$ cases (Table 3). The Paris classification of the cases, TACI mutation type, and complication are presented in Table 4.

Cases with complications and the cases without complications were compared, and also the cases with pulmonary complications were compared with lymphocyte counts, CD19, naive, nonswitch and switch B cell levels within the group. No statistically significant difference was found between the 2 groups (Table 5). When the cases with pulmonary complications were compared within the group, the CD8 ratio was high but the IgA level was low in patients with bronchiectasis compared to patients with other pulmonary complications, and CD3 was numerically

Table 2A. Lymphocyte and B cell values in the patients and in the control group

\begin{tabular}{|c|c|c|c|c|c|c|}
\hline \multirow{2}{*}{$\begin{array}{l}\text { Absolute } \\
\text { count }\end{array}$} & \multicolumn{2}{|c|}{ 4-8 years } & \multicolumn{2}{|c|}{ 8-12 years } & \multicolumn{2}{|c|}{ 12-18 years } \\
\hline & Patient & Control & Patient & Control & Patient & Control \\
\hline$n$ & 14 & 12 & 6 & 7 & 8 & 4 \\
\hline Lymphocyte & $4299 \pm 2289.3$ & $3315.8 \pm 779.8$ & $3227 \pm 1297.5$ & $2557.1 \pm 647.1$ & $3744.2 \pm 3049.1$ & $2090 \pm 183.8$ \\
\hline$p$ & \multicolumn{2}{|c|}{$>0.05$} & \multicolumn{2}{|c|}{$>0.05$} & \multicolumn{2}{|c|}{$>0.05$} \\
\hline CD19 & $1298.1 \pm 1401.9$ & $541.4 \pm 257.1$ & $571.7 \pm 141.5$ & $311 \pm 94.9$ & $570.7 \pm 603.4$ & $258.1 \pm 24.1$ \\
\hline$p$ & \multicolumn{2}{|c|}{$>0.05$} & \multicolumn{2}{|c|}{$<0.05$} & \multicolumn{2}{|c|}{$>0.05$} \\
\hline Naive & $576.4 \pm 307.9$ & $334 \pm 177.8$ & $415 \pm 135.8$ & $189.9 \pm 53.5$ & $447.2 \pm 551.5$ & $184.3 \pm 42.5$ \\
\hline$p$ & \multicolumn{2}{|c|}{$>0.05$} & \multicolumn{2}{|c|}{$<0.05$} & \multicolumn{2}{|c|}{$>0.05$} \\
\hline Non-switch & $26.1 \pm 29.2$ & $8.8 \pm 4.7$ & $15.3 \pm 5.8$ & $7.6 \pm 6.6$ & $24.4 \pm 30.6$ & $3 \pm 1.7$ \\
\hline$p$ & \multicolumn{2}{|c|}{$<0.05$} & \multicolumn{2}{|c|}{$>0.05$} & \multicolumn{2}{|c|}{$>0.05$} \\
\hline Switch & $85.5 \pm 77.6$ & $83.6 \pm 38.7$ & $54.2 \pm 50.5$ & $58 \pm 45.4$ & $99.2 \pm 181.4$ & $30.3 \pm 19.1$ \\
\hline$p$ & \multicolumn{2}{|c|}{$>0.05$} & \multicolumn{2}{|c|}{$>0.05$} & \multicolumn{2}{|c|}{$>0.05$} \\
\hline
\end{tabular}

Table 2B. Lymphocyte and B cell values in the patients and in the control group

\begin{tabular}{|c|c|c|c|c|c|c|}
\hline \multirow[t]{2}{*}{ Percent } & \multicolumn{2}{|c|}{ 4-8 years } & \multicolumn{2}{|c|}{ 8-12 years } & \multicolumn{2}{|c|}{ 12-18 years } \\
\hline & Patient & Control & Patient & Control & Patient & Control \\
\hline$n$ & 14 & 12 & 6 & 7 & 8 & 4 \\
\hline Lymphocyte & $46.1 \pm 13$ & $38 . \pm 11.6$ & $38.3 \pm 12$ & $38.9 \pm 12$ & $40.9 \pm 39.4$ & $29 \pm 5.3$ \\
\hline$p$ & \multicolumn{2}{|c|}{$>0.05$} & \multicolumn{2}{|c|}{$>0.05$} & \multicolumn{2}{|c|}{$>0.05$} \\
\hline CD19 & $21.6 \pm 9$ & $15.8 \pm 4.9$ & $17.7 \pm 5.3$ & $15.7 \pm 10$ & $13.3 \pm 7.7$ & $11.1 \pm 1.1$ \\
\hline$p$ & \multicolumn{2}{|c|}{$>0.05$} & \multicolumn{2}{|c|}{$<0.05$} & \multicolumn{2}{|c|}{$>0.05$} \\
\hline Naive & $71 \pm 9.8$ & $62.2 \pm 8.9$ & $72.4 \pm 12.1$ & $62.6 \pm 12.6$ & $63.9 \pm 29.6$ & $62.9 \pm 15.3$ \\
\hline$p$ & \multicolumn{2}{|c|}{$>0.05$} & \multicolumn{2}{|c|}{$>0.05$} & \multicolumn{2}{|c|}{$>0.05$} \\
\hline Non-switch & $2.8 \pm 2.1$ & $1.7 \pm 0.7$ & $2.9 \pm 1.4$ & $2.5 \pm 2.5$ & $3 \pm 2.9$ & $1.4 \pm 0.7$ \\
\hline$p$ & \multicolumn{2}{|c|}{$<0.05$} & \multicolumn{2}{|c|}{$>0.05$} & \multicolumn{2}{|c|}{$>0.05$} \\
\hline Switch & $11.1 \pm 6.4$ & $15.6 \pm 8.6$ & $10.9 \pm 7.2$ & $16.9 \pm 8.9$ & $10.6 \pm 11.5$ & $18.1 \pm 11.9$ \\
\hline$p$ & \multicolumn{2}{|c|}{$>0.05$} & \multicolumn{2}{|c|}{$>0.05$} & \multicolumn{2}{|c|}{$>0.05$} \\
\hline
\end{tabular}


Table 3. Complications seen in patients and their rates

\begin{tabular}{|c|c|c|}
\hline Complication & $n$ & Percent \\
\hline Entire complication* & 15 & 53.5 \\
\hline Pulmonary complications & 13 & 46.4 \\
\hline Bronchiectasis & 3 & 10.7 \\
\hline Asthma & 7 & 25 \\
\hline ILD & 3 & 10.7 \\
\hline Inflammatory bowel disease & 3 & 10.7 \\
\hline Lymphoproliferation & 3 & 10.7 \\
\hline Resistant parasitosis & 4 & 14.3 \\
\hline Growth retardation & 4 & 14.3 \\
\hline Autoimmunity & 1 & 3.6 \\
\hline
\end{tabular}

and proportionally low in the cases with ILD compared to others.

A 22-year-old patient died due to cor pulmonale and heart failure due to interstitial lung disease. The patients who had frequent and severe infections and severe complications (such as bronchiectasis) were administered with IVIG replacement at regular intervals determined according to their IgG levels (400-600 mg/kg). A total of 24 $(85.7 \%)$ patients are still being administered with IVIG replacement. 3 of 28 patients are followed with antibiotic prophylaxis, and 1 patient died. Three $(10.7 \%)$ of the patients receive steroid and mucolytic and additional antibiotic prophylaxis treatments for bronchiectasis and calcium, steroid, vitamin D treatments for the other complications, 7 patients $(25 \%)$ receive inhale steroid and/or montelukast treatment for asthma. One patient (3.6\%) uses colchicine due to the MEFV gene M694V heterozygote mutation. 3 patients $(10.7 \%)$ use steroids due to interstitial lung disease. However, rituximab and azathioprine treatment was initiated in one of them due to the development of relapse despite steroids, cyclosporin treatment was applied in addition to steroids in 1 patient. One of the patients (3.6\%) uses azathioprine due to inflammatory bowel disease.

\section{Discussion}

CVID is a complex disease in which immunologic defects and their clinical outcomes are highly variable. It is stated that the disease peaks in 2 different age groups including 2-5 years and 16-20 years. The age of diagnosis is accepted as minimum 4 years provided that all other immunological disorders are excluded $[1,2]$. In our study, it was seen that the average age of diagnosis of our patients was $80 \pm 44$ months (median: 71 months) and their symptom age was $45 \pm 45$ months (median: 36 months). These values correspond to the $1^{\text {st }}$ peak value of the diagnosis age and disease indicated in the literature $[1,2]$. When the patients are evaluated by gender distribution, it is seen that male gen- der is apparently in the forefront (68\% male, $32 \%$ female). Although the number of patients is low, this finding is not consistent with the literature indicating that both genders are equally affected [1, 4, 7-9]. When the family histories of our cases were examined, it was seen that $10.7 \%$ of them had family histories including a cousin and sibling followed with the diagnosis of CVID in the first-degree relative of 2 cases $(7.2 \%)$ and a sibling followed with a preliminary diagnosis of infant transient hypogammaglobulinemia in 1 case $(3.6 \%)$. This ratio was also reported as $32 \%$ in the study carried out by Llobet et al. [20] on Spanish children. The kinship ratio is $35.7 \%(10 / 28)$ in our cases. Mohammadinejad et al. [21] reported that $72 \%$ of 69 CVID cases had kinship. Karakoc-Aydiner et al. [22] reported that the kinship ratio was $75 \%$ in 20 CVID cases. Rivoisy et al. [23] reported that 24 of 436 CVID patients they reported in France had kinship. As it is seen, kinship ratios are different in different parts of our country, and also the kinship ratios reported from different countries are also different.

When presenting symptoms were evaluated, it was determined that the frequent infection was the presenting symptom in all of our cases. This is much more than the values of $20-30 \%$ given in the literature [1]. When Yong et al. [24] evaluated pediatric CVID patients, they determined the infection ratio as $38-50 \%$. However, in many literature data, the age group also includes the adults, and there are more different presentations such as lymphoproliferation, autoimmunity and rheumatic disease in the adult age group. While there was 1 patient with developed autoimmunity during follow-up in our cases, there was no patient presenting with autoimmunity. In other studies, the admission ratio with non-infectious findings is reported to be about $75 \%[25,26]$. This result, as it is interpreted in the literature, gives rise to the thought that autoimmunity and other symptoms emerge at older ages in CVID cases and the infections undergone with immunodeficiency convert balance in favor of autoimmunity [1, 2]. A great majority of infections detected in our cases are sinopulmonary infections, and gastrointestinal infections are less common.

The disruption of B cells in conversion to plasma cells and antibody production underlies the pathogenesis in CVID. At which subgroup level B-cell differentiation stops can be shown with the flow-cytometric techniques in the best way, and this approach is also the basis for CVID classification. In the literature, it is also seen that B cell count is affected only in $5-10 \%$ of CVID patients $[1,2,27]$. In our cases, lymphocyte counts, naive, nonswitch memory B cells were found to be statistically higher when compared to the control group proportionally and numerically $(p<0.05)$, switch (CD19+IgD-27+) memory B cells were found to be proportionally low but no statistical difference was determined. The switch memory B-cell ratios of our patients were $70 \%$ below the control group according to the ESID CVID diagnostic criteria. However our cases had obvious lymphocytosis. The number of lymphocytes in control sam- 
Table 4. Distribution of the patients according to the Paris classification, complications observed and TACI mutation results

\begin{tabular}{lllc}
\hline Patient & Paris & TACI & Complication \\
\hline Z.C.. & MB0 & No mutation & Asthma \\
\hline U.T. & MB2 & No mutation & Asthma \\
\hline B.K. & MB0 & No mutation & $\begin{array}{c}\text { ILD } \\
\text { Resistant Cryptococcus }\end{array}$ \\
& & & $\begin{array}{c}\text { Splenomegaly, cytopenia } \\
\text { Lymphoproliferation } \\
\text { Growth retardation }\end{array}$ \\
\hline D.A. & MB0 & & ILD \\
& & No mutation & IBD \\
& & & Growth retardation \\
& MB0 & & Splenomegaly, cytopenia \\
R.K. & MB0 & Resistant Giardia \\
\hline AS.Ş. & MB2 & No mutation & Asthma \\
\hline SN.Y. & MB2 & No mutation & Bronchiectasis \\
\hline HE.E. & MB2 & No mutation & Asthma \\
\hline M.T. & & No mutation & Asthma \\
& MB0 & No mutation & Bronchiectasis \\
IBD
\end{tabular}

\begin{tabular}{llll}
\hline T.G. & MB1 & No mutation & Asthma \\
\hline A.E. & MB0 & No mutation & \\
\hline B.A. & MB2 & No mutation \\
\hline N.K. & MB0 & No mutation \\
\hline
\end{tabular}

\begin{tabular}{llc}
\hline I.A. & MB2 & Positive (c.515 G>A(p.C172Y) homozygote) \\
\hline C.D. & MB2 & No mutation
\end{tabular}

Resistant Giardia

\begin{tabular}{|c|c|c|c|}
\hline R.T. & MB0 & Positive C104R heterozygote & Lymphoproliferation \\
\hline ME.T. & MB0 & No mutation & \\
\hline E.K. & MB2 & No mutation & \\
\hline P.A. & MB2 & Positive (heterozygote p.A181E(c.542C >A) & \\
\hline M.S. & MB2 & Positive (P21 Aheterozygote) & \\
\hline E.M. & MB2 & Positive (c.512 T>G(L171R) heterozygote) & \\
\hline S.Y. & MB0 & No mutation & \\
\hline B.S. & MB2 & No mutation & \\
\hline E.O. & MB1 & Positive (c.579C >A(p.C193X) homozygote) & \\
\hline K.H. & MB1 & Positive (heterozygote C298 ihsT(pC100Lfs'6) & \\
\hline
\end{tabular}


Table 5. Comparison of the patients with complications with the patients without complications and within the group

\begin{tabular}{|c|c|c|c|c|c|}
\hline & Lymphocyte count & B cell & Naive & Non-switch & Switch \\
\hline $\begin{array}{l}\text { Patients with complications } \\
\left(n=14^{*}\right)\end{array}$ & $4079.3 \pm 2685.3$ & $1089.9 \pm 1387.8$ & $563.3 \pm 450.5$ & $27.1 \pm 35.3$ & $103 \pm 151.4$ \\
\hline $\begin{array}{l}\text { Patients without complications } \\
(n=13)\end{array}$ & $3844.7 \pm 2042.2$ & $800.1 \pm 765.5$ & $444.6 \pm 287.7$ & $19.3 \pm 11.6$ & $62.3 \pm 41.8$ \\
\hline$p$ & 0.795 & 0.492 & 0.425 & 0.457 & 0.361 \\
\hline
\end{tabular}

ples from healthy children was also higher than shown in the literature. Especially in the 4-8 year-old group, frequent viral infections may be responsible for lymphocyte levels including switch memory. Vaccine response was evaluated as positive in $64.3 \%(18 / 28)$ of the patients and as negative in $35.7 \%(10 / 28)$ of the patients. Goldacker et al. reported that they received a positive response to polypeptide vaccines by $23 \%$ and to polysaccharide vaccines by $18 \%$, and there was no vaccine response in the rest of the cases [28].

Although CVID manifests mendelian inheritance in some families, it is generally thought to be a polygenic disease. As a result of their studies, EuroClass group [27] and Park et al. [2] suggest the investigation of ICOS, TACI, BAFF-R and CD19, with four proven monogenic defects leading to CVID at the final diagnosis stage. Only TACI mutation could be examined in our patients. The incidence of TACI mutation is reported to be approximately $10 \%$ in all CVID patients [29-31]. TACI mutation was present in $25 \%$ of our patients $(n=7)$, and this ratio was considerably higher compared to the literature. TACI mutation was homozygote positive in our 2 cases. Recent studies indicate that patients with TACI defects are more prone to lymphoproliferation and autoimmunity [29, 30]. Recurrent benign lymphoproliferation was present in one of our patients with positive TACI mutation. However, autoimmunity did not develop during the follow-up in any of them.

Maarschalk-Ellerbroek et al. [17] report that $51 \%$ of 47 CVID cases with an average age of 37 had no complications, and complications such as autoimmunity, lymphoproliferation, and enteropathy, especially pulmonary complications are seen in the rest of them. Rivoisy et al. [23] reported that $62.5 \%$ of 436 CVID patients they reported from France had splenomegaly, $29 \%$ of them had granulomatous diseases, and $58 \%$ of them had bronchiectasis.

Chronic lung disease is the most common complication in CVID patients and affects $29-58 \%$ of patients [17, 23, 32]. The pulmonary complications observed in the follow-up of the patients with CVID are asthma and chronic obstructive pulmonary disease, ILD and structural airway disease (airway wall thickening, air trapping, and bronchiectasis). Pulmonary complications are the most important cause of mortality and morbidity in these patients. The pulmonary complication was observed in $46.4 \%$ (13/28) of our cases. Chase et al. [33] reported that granulomatous and lymphocytic interstitial lung disease (GLILD) developed in 10-15\% of CVID patients, and this was often accompanied by splenomegaly, lymphadenopathy, autoimmune cytopenia, gastrointestinal and hepatic disease. Maarschalk-Ellerbroek et al. [17] report airway disease at $30 \%$ and ILD at $34 \%$. The prevalence of bronchiectasis in patients with CVID is reported to be $29-71 \%$ [34, 35]. In our study, asthma occurred in $25 \%$, bronchiectasis in $10.7 \%$ and ILD in $10.7 \%$ of participants. Yong et al. [24] detected bronchiectasis in $4 \%$ of the cases and asthma in $29 \%$ of the cases. Bronchiectasis is a consequence of recurrent lower respiratory tract infections and is an important cause of morbidity and mortality, especially in CVID patients $[1,17,32]$. The lower presence of pulmonary complications in our patients is pleasing in terms of the quality of follow-up. Another complication seen in the lungs is nodules. Nodules were observed in the lungs in three of our cases $(10.7 \%)$. This ratio is consistent with the results of the nodule and granuloma presence given as $5-10 \%$ and $10-22 \%$, respectively, in the literature [12, 24, 35]. The CD8 ratio was high but the IgA level was low in patients with bronchiectasis compared to patients with other pulmonary complications, and CD3 was numerically and proportionally low in the cases with ILD compared to others. No evidence to support these findings was found in the literature. Therefore, we thought that a better comparison could be made with more patients.

The gastrointestinal system and lungs are the regions where granulomas are most frequently observed, and skin, spleen, and liver involvement is also reported in the literature. The incidence of these non-caseating granulomas, especially similar to sarcoidosis, is given at $8-22 \%[12,25,36]$. Three of our patients had common nodules in the lungs, and lymphocyte accumulation was detected in the biopsy performed, and it was evaluated as lymphoproliferation. Lymphoproliferation is accompanied by inflammatory bowel disease, growth retardation and splenomegaly in one of these patients, by discoid lupus in the other one, and by splenomegaly in the third patient.

Resistant Giardia infection was observed in three cases. In 3 cases, it was determined that the biopsies performed due to ongoing diarrhea were compatible with inflammatory bowel disease. It is accompanied by resistant cryptococcus infection in one case. When the literature was examined, chronic diarrhea and malabsorption were reported in approximately $50 \%$ of the patients, they were mostly stated to be secondary to infection [1, 2, 37-39]. In the evaluation made by Yong et al. [24] in 45 pediatric CVID patients, they noted gastrointestinal 
system findings (malabsorption, celiac disease, lymphoid hyperplasia, inflammatory bowel disease) in $16 \%$ of the cases, Bezronik et al. [40] found them in $19 \%$ of the cases in their study on 31 pediatric and adult patients. Our ratios are lower when compared to both adults and other pediatric studies. This may also be a consequence of the earlier diagnosis and earlier initiation of the treatment.

Autoimmune-related diseases (autoimmune cytopenia, vasculitis, relapsing polychondritis, seronegative spondyloarthropathy) have been increasingly associated with CVID in recent years. The autoimmunity ratio was $25 \%$ in pediatric group studies, discoid lupus development was observed in 1 patient (3.6\%) during the follow-up in our study. While only the presence of autoimmune cytopenia is given as $5-8 \%$ in the literature, no autoimmune cytopenia was detected in our patients, and hypersplenism-associated cytopenia developed in 2 of our patients [12, 35-37, 41, 42].

Upon evaluating lymphoproliferation and malignancy, benign lymphoproliferation was observed in 3 of our patients $(10.7 \%)$. In the literature, it is reported that marginal zone lymphoma (MZL), which is a mucosa-associated type of lymphoma, which is separated from the lymphoma group in the WHO's latest classification and is mostly attributed to CVID group patients, is observed. Furthermore, small cell non-Hodgkin's lymphoma is most frequently observed among lymphomas. Hodgkin's lymphoma is rarely seen and has only been reported on a case-by-case basis. Although the incidence of lymphoma is given as about $2-8 \%$ in the large patient series in the literature, Hodgkin's lymphoma was not observed in our patients, and neither was other malignancy type [1, 2, 24, $37,43,44]$.

Splenomegaly was detected in four of our cases (14.3\%). Various studies carried out on CVID have shown the relationship of splenomegaly with the underlying immunological defect. In particular, it has been argued that B cell subgroup development continues in the peripheral lymph node and spleen, and clinical findings are accompanied by splenomegaly in disorders developing there $[2,12,26,27]$.

When our cases were compared with the cases with and without developed complications, no statistical difference was observed in terms of the total lymphocyte and B memory cell profile between the 2 groups. 8 (53.3\%) of 15 cases with complications were MB0, 1 (7.1\%) of them was MB1, and 6 (42.9\%) of them were MB2. 3 of 8 cases with MB0 had splenomegaly and inflammatory bowel disease, and severe complications were observed at higher rates compared to the other groups. This finding was also consistent with the literature [19, 27]. 2 of 3 cases in which lymphoproliferation was observed and 2 of 3 cases in which ILD was observed were MB0. These findings were similar to the previous literature data [19].

IVIG replacement constitutes the basis of the treatment. The aim is to protect against life-threatening serious bacterial infections or to reduce the frequency of these infections $[10,34,45]$. Dolcino et al. [45] emphasized that the IVIG replacement treatment has immunomodulatory effects in CVID patients, and it is necessary to carry out research not only to prevent infections but also on the timing of the IVIG treatment due to its immunomodulatory effects.

\section{Conclusions}

When the patients with CVID, firstly, had been compared to controls, and then those with CVID-related complications had been compared to the ones without, no statistical difference was found. Thus, we concluded that diagnostic approach is limited due to the absence of apparent objective data, although the effect in B cells during transition from non-switch to switch memory phase is thought to be a functional influence. Nevertheless, we observed changes in CD3 and CD8 cells but B-cell based classification is not enough for differential diagnosis and the follow-up of disease prognosis. We believe that new and more comprehensive flow cytometric and genetic investigations are required.

According to the literature, TACI mutation is reported in $7-21 \%$ of CVID cases. But in our cases this rate was much higher. We believe that the clinical manifestation of this mutation may be variable, such as lymphoproliferation, autoimmunity, hypogammaglobulinemia, and may be more positive among the immunodeficiency patients, even at higher rates within the community. We may also think that this genetic allele frequency may be high in our own societies. Comprehensive genetic screening studies are essential for this reason.

The authors declare no conflict of interest.

\section{References}

1. Chapel H, Cunningham-Rundles C (2009): Update in understanding common variable immunodeficiency disorders (CVIDs) and the management of patients with these conditions. BJH Review 145: 709-727.

2. Park MA, Li JT, Hugan JB, et al. (2008): Common variable immunodeficiency: a new look at an old disease. Lancet 372: 489-502.

3. Notarangelo LD (2010): Primary Immunodeficiencies. J Allergy Clin Immunol 125: 182-194.

4. Pan-Hammarström Q, Hammarström L (2008): Antibody deficiency diseases. Eur J Immunol 38: 327-333.

5. Schroeder HW Jr. (2000): Genetics of IgA deficiency and common variable immunodeficiency. Clin Rev Allergy Immunol 19: 127-140.

6. Yazdani R, Hakemi MG, Sherkat R, et al. (2014): Genetic defects and the role of helper T-cells in the pathogenesis of common variable immunodeficiency. Adv Biomed Res 3: 2.

7. Bonilla FA, Berstein IL, Khan DA, et al. (2005): Practice parameter for the diagnosis and management of primary immunodeficiency. Ann Allergy Asthma Immunol 94: S1-63.

8. Fasth A (1982): Primary immunodeficiency disorders in Sweden: cases among children, 1974-1979. J Clin Immunol 2: 86-92.

9. McCluskey DR, Boyd NAM (1989): Prevalence of primary hypogammaglobulinemia in Northern Ireland. Proc R Coll Physicians Edinb 19: 191-194. 
10. Kutukculer N, Gulez N, Karaca NE, et al. (2012): Three Different Classifications, B Lymphocyte Subpopulations, TNFRSF13B (TACI), TNFRSF13C (BAFF-R), TNFSF13 (APRIL) Gene Mutations, CTLA-4 and ICOS Gene Polymorphisms in Turkish Patients with Common Variable Immunodeficiency. J Clin Immunol 32: 1165-1179.

11. ESID Registry - Working definitions for clinical diagnosis of PID March 5, 2014. https://esid.org/

12. Cunningham-Rundles C, Bodian C (1999): Common variable immunodeficiency: clinical and immunological features of 248 patients. Clin Immunol 92: 34-48.

13. Hammarström L, Vorechovsky I, Webster D (2000): Selective IgA deficiency (SigAD) and common variable immunodeficiency (CVID). Clin Exp Immunol 120: 225-231.

14. Vorechovsky I, Zetterquist H, Paganelli R, et al. (1995): Family and linkage study of selective IgA deficiency and common variable immunodeficiency. Clin Immunol Immunopathol 77: 185192.

15. Salzer U, Warnatz K, Peter HH (2012): Common variable immunodeficiency - an update. Arthritis Res Ther 14: 223.

16. Sekine H, Ferraire RC, Pan-Hammarström Q, et al. (2007): Role for Msh5 in the regulation of Ig class switch recombination. Proc Natl Acad Sci USA 104: 7193-7198.

17. Maarschalk-Ellerbroek LJ, Van Montfrans JM, Lammers JWJ, et al. (2014): CT Screening for Pulmonary Pathology in Common Variable Immunodeficiency Disorders and the Correlation with Clinical and Immunological Parameters. J Clin Immünol 34: 642-654.

18. Aksu G, Genel F, Koturoglu G, et al. (2006): Serum immunoglobulin (IgG, IgM, IgA) and IgG subclass concentrations in healthy children: a study using nephelometric technique. Turk J Pediatr 48: 19-24.

19. Piqueras B, Lavenu-Bombled C, Galicier L, et al. (2003): Common variable immunodeficiency patient classification based on impaired B cell memory differentiation correlates with clinical aspects. J Clin Immunol 23: 385-400.

20. Llobet MP, Soler-Placin P, Detkova D, et al. (2009): Common variable immunodeficiency: 20-yr experience at a single centre. Pediatr Allergy Immunol 20: 113-118.

21. Mohammadinejad P, Aghamohammadi A, Abolhassani H, et al. (2012): Pediatric Patients With Common Variable Immunodeficiency: Long-term Follow-up. J Investig Allergol Clin Immunol 22: 208-214.

22. Karakoc-Aydiner E, Ozen AO, Baris S, et al. (2014): Alteration in Humoral Immunity Is Common Among Family Members of Patients With Common Variable Immunodeficiency. J Investig Allergol Clin Immunol 24: 346-351.

23. Rivoisy C, Gérard L, Boutboul D, et al. (2012): Parental consanguinity is associated with a severe phenotype in common variable immunodeficiency. J Clin Immunol 32: 98-105.

24. Yong PL, Orange JS, Sullivan KE (2010): Pediatric common variable immunodeficiency: Immunologic and phenotypic associations with switched memory B cells. Pediatr Allergy Immunol 21: 852-858.

25. Salzer U, Grimbacher B (2006): Common variable immunodeficiency: the power of co-stimulation. Semin Immunol 18: 337-346.

26. Cunningham-Rundles C, Bodian C (1999): Common variable immunodeficiency: clinical and immunological features of 248 patients. Clin Immunol 92: 34-48.

27. Wehr C, Kivioja T, Schmitt C, et al. (2008): The EUROclass trial: defining subgroups in common variable immunodeficiency. Blood 111: 77-85.
28. Goldacker S, Draeger R, Warnatz K, et al. (2007): Active vaccination in patients with common variable immunodeficiency (CVID). Clin Immunol 124: 294-303.

29. La Cava A (2013): Common variable immunodeficiency: two mutations are better than one. J Clin Invest 123: 4142-4143.

30. Tam JS, Routes JM (2013): Common variable immunodeficiency. Am J Rhinol Allergy 27: 260-265.

31. Abolhassani H, Parvaneh N, Rezaei N, et al. (2014): Genetic Defects in B-Cell Development and Their Clinical Consequences. J Investig Allergol Clin Immunol 24: 6-22.

32. Maglione PJ, Overbey JR, Radigan L, et al. (2014): Pulmonary radiologic findings in common variable immunodeficiency: clinical and immunological correlations. Ann Allergy Asthma Immunol 113: 452-459.

33. Chase NM, Verbsky JW, Hintermeyer MK, et al. (2013): Use of Combination Chemotherapy for treatment of Granulomatous and Lymphocytic Interstitial Lung Disease (GLILD) in Patients with Common Variable Immunodeficiency (CVID). J Clin Immunol 33: 30-39.

34. Van DV, Van Montfrans JM, Terheggen-Lagro SW, et al. (2010): ACT Scan Score for the Assessment of Lung Disease in Children With Common Variable Immunodeficiency Disorders. Chest 138: 371-379.

35. Giannouli S, Anagnostou D, Soliotis F, Voulgarelis M (2004): Autoimmune manifestations in common variable immunodeficiency. Clin Rheumatol 23: 449-452.

36. Brandt D, Gershwin ME (2006): Common variable immunodeficiency and autoimmunity. Autoimmun Rev 5: 465-470.

37. Ardeniz O, Cunningham-Rundles C (2009): Granulomatous disease in common variable immunodeficiency. Clin Immunol 133: 198-207.

38. Daniels JA, Lederman HM, Maitra A, Montgomery EA (2007): Gastrointestinal tract pathology in patients with common variable immunodeficiency (CVID): a clinicopathologic study and review. Am J Surg Pathol 31: 1800-1812.

39. Chapel H, Lucas M, Lee M, et al. (2008): Common variable immunodeficiency disorders: division into distinct clinical phenotypes. Blood 112: 277-286.

40. Bezronik L, Carelli D, Giovanni DD, et al. (2010): Common variable immunodeficiency retrospective study in 31 Argentine patients. XIV: Meeting of the ESID 73.

41. Hermaszevsi RA, Webster AD (1993): Primary hypogammaglobulinemia: a survey of clinical manifestations and complications. Q J Med 86: 31-42.

42. Haymore BR, Mikita CP, Tsokos GC (2008): Common variable immune deficiency (CVID) presenting as an autoimmune disease: role of memory cells. Autoimmun Rev 7: 309-312.

43. Chua I, Quinti I, Grimbacher B (2008): Lymphoma in common variable immunodeficiency: interplay between immune dysregulation, infection and genetics. Curr Opin Hematol 15: 368-374.

44. Mellemkjaer L, Hammarstrom L, Andersen V, et al. (2002): Cancer risk among patients with IgA deficiency or common variable immunodeficiency and their relatives: a combined Danish and Swedish study. Clin Exp Immunol 130: 495-500.

45. Dolcino M, Patuzzo G, Barbieri A, et al. (2014): Gene Expression Profiling in Peripheral Blood Mononuclear Cells of Patients with Common Variable Immunodeficiency: Modulation of Adaptive Immune Response following Intravenous Immunoglobulin Therapy. PLoS One 9: e97571. 